\title{
Interpolated Derivatives
}

By B. SpatN

(Received 21st July, 1955.)

In a previous paper [Spain, Proc. Roy. Soc. Edinburgh, Vol. LX (1940), 134], I have shown that the application of the cardinal function to the problem of interpolating the derivatives yields the result

$$
D^{n} f(x)=\frac{1}{\Gamma(-n)} \int_{a}^{x} \frac{f(u) d u}{(x-u)^{n+1}}+\frac{1}{\Gamma(-n)} \int_{-\infty}^{a} d u f(u) \int_{1}^{\infty} \frac{t^{n} e^{-l(x-u)} d t}{\Gamma(n+1)} .
$$

This formula is valid for $x>a$ (the constant of integration), and $R(n)<0$. The analytical continuation for $R(n) \geqslant 0$ is indicated in the paper just quoted. The first term is the familiar expression for a fractional derivative, but the second term is not Riemann's complementary function. Furthermore, this result is unsatisfactory because it is impossible to perform the repeated operation of a fractional derivative of a fractional derivative.

The cardinal function interpolation requires the derivatives to be given for both positive and negative values of $n$. Instead let us try and interpolate by the Gregory-Newton formula for negative values of $n$. The sth repeated integral of $f(x)$ can be written

$$
F(s)=\frac{1}{(s-1) !} \int_{a}^{x}(x-u)^{s-1} f(u) d u .
$$

The Gregory-Newton formula is

$$
F(n)=\sum_{r=0}^{\infty} \frac{n(n-1) \ldots(n-r+1)}{r !} \Delta^{r} F
$$

where

$$
\Delta^{r} F=\sum_{s=0}^{r}(-1)^{r+s}\left(\begin{array}{l}
r \\
s
\end{array}\right) F(b+w s)
$$

and $F(n)$ the function to be interpolated is given at the points $b, b+w, \ldots, b+w s, \ldots$ In our problem $w=b=1$ and so $b+w s=1+s$ and by substitution we have

$$
\begin{aligned}
F(n+1) & =\sum_{r=0}^{\infty} \frac{n(n-1) \ldots(n-r+1)}{r !} \sum_{s=0}^{r}(-1)^{r+s}\left(\begin{array}{l}
r \\
s
\end{array}\right) \frac{1}{s !} \int_{a}^{x}(x-u)^{s} f(u) d u \\
& =\sum_{r=0}^{\infty} \frac{(-1)^{r} n(n-1) \ldots(n-r+1)}{r !} \int_{a}^{x} d u f(u) \sum_{s=0}^{r}(-1)^{s}\left(\begin{array}{l}
r \\
s
\end{array}\right) \frac{(x-u)^{s}}{s !} \\
& =\sum_{r=0}^{\infty} \frac{(-1)^{r} n(n-1) \ldots(n-r+1)}{r !} \int_{a}^{x} f(u) L_{r}(x-u) d u \\
& =\int_{a}^{x} d u f(u) \sum_{r=0}^{\infty} \frac{(-1)^{r} n(n-1) \ldots(n-r+1)}{r !} L_{r}(x-u),
\end{aligned}
$$


where $L_{r}(x)$ is the normalised Laguerre polynomial of degree $r$ (Kaczmarz and Steinhaus, Theorie der Orthogonalreihen, 140) defined by

$$
L_{r}(x)=\frac{1}{r !} e^{x} \frac{d^{r}}{d x^{r}}\left(e^{-x} x^{r}\right)
$$

with the orthogonal properties

$$
\int_{0}^{\infty} e^{-x} L_{r}(x) L_{l}(x) d x=\left\{\begin{array}{l}
0 \text { if } r \neq t \\
1 \text { if } r=t .
\end{array}\right.
$$

The summation we require is readily obtained if we expand $x^{n}$ for nonintegral $n>0$ in a series of Laguerre polynomials. That is, write

$$
x^{n}=\sum_{r=0}^{\infty} \lambda_{r} L_{r}(x)
$$

and by the orthogonal properties of the Laguerre polynomials we have

$$
\lambda_{r}=\int_{0}^{\infty} e^{-x} x^{n} L_{r}(x) d x=\frac{1}{r !} \int_{0}^{\infty} x^{n} \frac{d^{r}}{d x^{r}}\left(e^{-x} x^{r}\right) d x .
$$

Since $n>0$, repeated application of integration by parts yields

$$
\begin{aligned}
\lambda_{r} & =\frac{(-1)^{r} n(n-1) \ldots(n-r+1)}{r !} \int_{0}^{\infty} x^{n-r}\left(e^{-x} x^{r}\right) d x \\
& =\frac{(-1)^{r} n(n-1) \ldots(n-r+1) \Gamma(n+1)}{r !} .
\end{aligned}
$$

Thus

$$
\frac{x^{n}}{\Gamma(n+1)}=\sum_{r=0}^{\infty} \frac{(-1)^{r} n(n-1) \ldots(n-r+1)}{r !} L_{r}(x)
$$

and so

$$
F(n+1)=\frac{1}{\Gamma(n+1)} \int_{a}^{x}(x-u)^{n} f(u) d u .
$$

That is,

$$
D^{-n} f(x)=\frac{1}{\Gamma(n)} \int_{a}^{x}(x-u)^{n-1} f(u) d u .
$$

We see that the Gregory-Newton interpolation formula does give the familiar generalisation of the fractional derivative.

Sir John Cass College,

LONDON. 